\title{
N94-10570
}

\section{ONE DIMENSIONAL REPRESENTATIONS IN QUANTUM OPTICS}

\author{
J. Janszky, P. A dam, I. Földesi \\ Research Laboratory for Cryslal Phyoico \\ PO Bos 13l, H.150e Budapest, Hungary \\ An. V. Vinogradov \\ Lebedev Inotilute of Physics, Moscov, Rresia
}

\begin{abstract}
A bstract
The possibility to represent the quantum states of a harmonic oscillator not on the whole a-plane but on its one dimensional manifolds is considered. It is shown that a simple Gaussian distribution along a straight line describes a quadrature squeesed state while a similar Gaussian distribution along a circle leads to the amplitude squeesed state. The connection between the one dimensional representations and the usual Glauber representation is discussed.
\end{abstract}

\section{Introduction}

There are several widely used representations to describe a state of a quantum oscillator in the Hilbert space. The most natural one is the expansion of the state into the number state

$$
\left|c>=\sum_{n=0}^{\infty} c_{n}\right| n>\text {. }
$$

A nother well known possibility is the coherent state representation $[1,2]$

$$
\left|f>=\frac{1}{\pi} \int f\left(\alpha^{*}\right) \exp \left(-|\alpha|^{2} / 2\right)\right| \alpha>d^{2} \alpha, d^{2} \alpha=d(R e \alpha) d(\operatorname{Im} \alpha),
$$

$f\left(\alpha^{*}\right)$ being an analytical function of $\alpha^{*}$. Here the state is represented by a superposition of nonorthogonal coherent states all over the complex $\alpha$-plane.

As already Glauber pointed out, there is an infinite number of ways of expanding any state in terms of coherent states due to the overcompleteness of the latter states

$$
\left|f>=\frac{1}{x} \int G\left(\alpha, \alpha^{*}\right)\right| \alpha>d^{2} \alpha,
$$

here the expansion function $G\left(\alpha, \alpha^{*}\right)$ may be a rather general function of $\alpha$ and $\alpha^{*}$. Being confined to some given class of functions the uncertainty in finding $G\left(\alpha, \alpha^{*}\right)$ can be reduced. In 
this paper we shall deal with such representations that correspond to kern fonctions $G\left(\alpha, \alpha^{*}\right)$ leading to integration over a one dimensional manifold of the $\alpha$-plane in Eq. (3). The possibility to represent any state on a subspace of the complex plane comes from Cahill's theorem on overcompleteness $[3,4 \mid$. We shall show that such nonclassical states as the quadrature and amplitude squeezed states can be represented very naturally by superposition of coherent states along a straight line or along a circle in the $\alpha$-plane correspondingly.

\section{Representation along a straight line}

The most simple states emerging from superposition of coherent states are the even $\left|x_{1}+\right\rangle$ and the odd $\mid x,->$ states

$$
\begin{aligned}
& \mid x_{1}+>=c_{+}(|x>+|-x>), \\
& \mid x_{1}->=c_{-}(|x>-|-x>),
\end{aligned}
$$

where $\mid x>$ is a usual coherent state with real eigenvalue of the annihilation operator $a \mid x>=$ $x \mid x>$. It is remarkable that the even state $\mid x,+>$ being a superposition of two classical states is squeered $|5|$

$$
\left(\Delta \varepsilon_{2}\right)^{2}=\frac{1}{4}-x^{2} /\left|1+\exp \left(2 x^{2}\right)\right|
$$

where 1 and $a_{2}$ are the Hermitian quadratures of the annihilation operator.

The squeexing can be further enhanced by adding the vacaum state to $\mid x_{1}+>$

$$
|x, p\rangle=c_{p}(|x\rangle+p|0\rangle+\mid-x>) \text {. }
$$

This way one can achieve a variance $\left(\Delta_{\alpha_{2}}\right)^{2}=0.0651$ instead of 0.111 for $\mid x_{1}+>$ or 0.25 for the vacu um state. Superposing more and more even states to it one can get even more squeezing

$$
\left|f>=\int_{-\infty}^{\infty} f(x)\right| x>d x .
$$

In fact for any positive even fanction $f(x)$, but for the $f(x)=\delta(x)$ describing the vacuum, the state defined by $\mathrm{Eq} .(8)$ is squeezed. A most important particular case is the Gaussian superposition function $[5,6]$

$$
f(x)=c \exp \left(-x^{2} / \gamma^{2}\right), \quad c=\sqrt{\sqrt{\left(1+\gamma^{2}\right)} / \gamma^{2} x},
$$

describing the osual squeezed vacuum state with uncertainties of the quadratures

$$
\left(\Delta a_{1}\right)^{2}=\left(1+\gamma^{2}\right) / 4, \quad\left(\Delta a_{2}\right)^{2}=1 / 4\left(1+\gamma^{2}\right) .
$$


Similar distributions can be constructed not only along the real axis but along any straight line. For example the squeezed coherent state with coherent signal $\alpha$ and squeezing parameter $\zeta=r \exp (i \theta)$ can be written in the form $|7,8|$

$$
\begin{gathered}
\left|\alpha, s>=\int_{-\infty}^{\infty} f(x, \alpha, s)\right| \alpha+\exp (i \theta / 2) x>d x, \\
\left.f(x, \alpha, s)=c \exp \left(-x^{2} / \gamma^{2}-i \delta s\right), \quad \delta=I m \mid \alpha^{*} \exp (i \theta / 2)\right], \quad \gamma=\sqrt{e^{2 r}-1} .
\end{gathered}
$$

As the Gaussian superposition of coherent states of Eq. $(8,0)$ was a useful generalisation of the even states of Eqs. $(4,7)$ analogously one can build an odd state $\mid \gamma, 1>$ resembling Eq. (5)

$$
\begin{gathered}
\left|\gamma, 1>=\int_{-\infty}^{\infty} G(x, \gamma, 1)\right| x>d x, \\
G(x, \gamma, 1)=c_{1} x \exp \left(-x^{2} / \gamma^{2}\right), \quad c_{1}=\frac{2}{\gamma^{3}} \sqrt{\sqrt{\left(1+\gamma^{2}\right)^{3}} / x} .
\end{gathered}
$$

The mean photon number and the uncertainty of the quadrature $a_{2}$ in this state are

$$
\left\langle\gamma, 1\left|a \dagger_{a}\right| \gamma, 1\right\rangle=1+\frac{3 \gamma^{4}}{4\left(1+\gamma^{2}\right)}
$$

and

$$
\left(\Delta \mathfrak{a}_{2}\right)^{2}=\frac{3}{4\left(1+\gamma^{2}\right)} .
$$

We can see that the state $|\gamma, 1\rangle$ coincides with the one photon state $|1\rangle$ in the limit $\gamma=0$ and with increasing $\gamma$ at $\left\langle\gamma, 1\left|a t_{a}\right| \gamma, 1\right\rangle=2, \gamma=\sqrt{2}$ it becomes squeered.

Similarlyone can define states $\mid \gamma, n>$ with $x^{n}$ instead of $x$ in their weight function $G(x, \gamma, n)$. Superpositions of such states leading to Hermite polynomial weight functions are rather remarkable

$$
\begin{gathered}
\left|h_{n}>=\int_{-\infty}^{\infty} h_{n}(x)\right| x>d x \\
h_{n}(x)=\sqrt{\sqrt{3} /(2 \pi n !)} H_{n}\left(\frac{\sqrt{3} x}{2}\right) \exp \left(-x^{2} / 2\right) .
\end{gathered}
$$

The states $\left|h_{n}\right\rangle$ are orthonormalized $\left(\left\langle h_{n} \mid h_{m}\right\rangle=\delta_{n m}\right)$, satisfy the relation

and correspondingly

$$
\text { a }\left|h_{n}\right\rangle=\sqrt{\frac{n+1}{3}}\left|h_{n+1}\right\rangle+\sqrt{\frac{4 n}{3}}\left|h_{n-1}\right\rangle
$$




$$
<h_{n}|a| h_{n}>=0,\left\langle h_{n}\left|a^{2}\right| h_{n}\right\rangle=\frac{2}{3}(2 n+1),\left\langle h_{n}\left|a^{\dagger}\right| h_{n}\right\rangle=\frac{5 n+1}{3} \text {. }
$$

The projection operator constructed from the Hermite states

$$
P_{h} \equiv \sum_{n=0}^{\infty}\left|h_{n}><h_{n}\right|
$$

is a unity operator both for the coherent and photon number states

$$
\begin{aligned}
&<x\left|P_{h}\right| y>=<x|y>=\exp |-(x-y)^{2} / 2 \mid, \\
&<n\left|P_{h}\right| m>=\delta_{n m},
\end{aligned}
$$

which shows that any state can be represented by them. For example one can expand a $\mid f>=$ $\int_{-\infty}^{\infty} f(x) \mid x>d x$ state into the $\mid h_{n}>$ states

$$
\left|f>=\sum_{n=0}^{\infty} f_{n}\right| h_{n}>,
$$

where

$$
\left.f_{n}=\iint_{-\infty}^{\infty} f(x) h_{n}(y) \exp \mid-(x-y)^{2} / 2\right] d x d y
$$

\section{Representation along a circle}

Let us now consider a state emerging from superposition of coherent states with the same amplitude $|\alpha|=R \mathrm{i}$. e. we choose only those coherent states which lie on the same circle in the $\alpha$-plane [5].

$$
\left|F, R>=\frac{\exp \left(R^{2} / 2\right)}{2 \pi} \oint F(\phi)\right| R \exp (i \phi)>d \phi .
$$

If the radius of the circle is chosen big enough so that $\mathrm{Eq}$. (2) can be replaced by

$$
\left|f>=\frac{1}{\pi} \int_{|\alpha|<R} f\left(\alpha^{*}\right) \exp \left(-|\alpha|^{2} / 2\right)\right| \alpha>d^{2} \alpha,
$$

then we can find connections between the distribution fonction $F(\phi)$ and Glauber's weight function $f\left(\alpha^{*}\right)$

$$
F(\phi)=\frac{R \exp (i \phi)}{\pi} \int_{|\alpha|<R} f\left(\alpha^{*}\right) \frac{\exp \left(-|\alpha|^{2}\right)}{z-\alpha} d^{2} \alpha, \quad z=R \exp (i \phi)
$$


and

$$
f\left(\alpha^{*}\right)=\oint F(\phi) \exp \left(\alpha^{*} z\right) d \phi
$$

We note that if one knows the time behaviour of the annihilation operator a $(t)$ the analytic expansion function $f\left(\alpha^{*}, t\right)$ can be foond from the expression $|\theta|$

$$
f\left(\alpha^{*}, l\right)=\int d^{2} \eta x(\eta, \ell) \exp \left(-|\pi|^{2}-q \alpha^{*}\right) / \sqrt{\pi \int d^{2} \eta x(\eta, \ell) \exp \left(-|\eta|^{2}\right)},
$$

where $\chi(\eta, 1)$ is the normally ordered characteristic fonction ( $p$ being the density operator)

$$
\chi(\eta, t)=\operatorname{Tr}\left|p \exp \left(q a^{\dagger}(t)\right) \exp \left(-q^{\star} a(t)\right)\right| .
$$

Using Eq. (28) we find for the n-photon state and the coherent state correspondingly

$$
\begin{gathered}
F(\phi, n)=\sqrt{n \mid R^{-n}} \exp (-i n \phi), \\
F(\phi, \alpha)=\frac{z \exp \left(-|\alpha|^{2} / 2\right)}{z-\alpha}, \quad|\alpha|<R .
\end{gathered}
$$

According to Eq. (32) we can obtain the coefficients of the $n$-photon representation $c_{n}$ of $E q$. (1) if we know the distribution function $F(\phi)$

$$
c_{n}=R^{n} F_{n} / \sqrt{n !},
$$

where $F_{n}$ are Fourier, coefficients of $F(\phi)$

$$
F(\phi)=\sum_{n=0}^{\infty} \exp (-i n \phi) F_{n} .
$$

An interesting state is the state with Gaussian distribution function | $y>$

$$
F(\phi, x)=c_{x} \exp \left(-i \delta \phi-\frac{x^{2}}{2} \phi^{2}\right) .
$$

In case of extremely large $\mathrm{a}$ it describes the usual coherent state while in the opposite limit it is the $n$-photon state $(n=\delta)$. Between these states it will be an amplitude squeezed banana state. Graphically it can be understood if one visualizes how with decreasing a the muffin-like coherent state going through a squeezed crescent-like state deforms along the circle into the donut-like number state.

It is also worth mentioning that the Gaussian superposition of coherent states along an arc are not only describe amplitude squeezing $\{10,11\}$ but they are also approximate number-phase intelligent states $|10|$ associated with the Pegg-Barnett phase operator |12].

Remarkble feature of this state is the complete analogy with the usual quadrature squeezed state discussed in the previous Section, as the Gaussian arc distribution is amplitude squeezed while the Gaussian straight line distribution is quadrature squeezed. Moreover, as the even 
superposition of two coherent states from $\mathrm{Eq}$. (4) can be derived by truncation from the straight line Gaussian state of Eqs. $(5,9)$ so Schleich's superposition state [13]

$$
\mid \varphi>=c_{\alpha \phi}\left(\left|\alpha e^{i \phi / 2}>+\right| \alpha e^{-i \phi / 2}>\mid\right. \text {, }
$$

similarly can be considered as a truncated arc Ganssian state of Eqs. $(26,36)$.

A physical example, the so called phonon squeezing [14], where an arc distributed state occurs is the Franck.Condon transition induced by short coherent light polse in a molecule $[6,14,15]$. It is worth mentioning that using Eq. (28) one can to some extent purposefully shape the molecular vibrational state by special choice of the characteristics of the exiting light pulse. For example we showed that by appropriate linear chirp the vibrational state can be turned in the $\alpha$-plane while using nonlinear chirp the amplitude squeesed vibrational state can be deformed into a typical quadrature squeeved form.

\section{Acknowledgments}

This work was supported by the National Research Foundation of Hungary under contract 1444.

\section{References}

[1] R. J. Glauber, Phys. Rev. 131, 2766 (1963).

|2| J. R. Klander and E. C. G. Sudarshan, Fundement als of Quantrm Optice, (W. A. Benjamin Inc., New York, 1968).

[3] K. E. Cahill, Phys. Rev. 138, B1566 (1965).

[4] A. Vourdas, Phys. Rev. A 41, 1653 (1990).

(5) J. Janseky and An. V. Vinogradov, Phys. Rev. Lett. 64, 2771 (1990).

(6) V. Buzek and P. L. Knight, Optics. Comm. 81, 331 (1991), V. Buzek, A. VidiellaBarranco and P. L. Knight, Phys. Rev. A 45, 6570 (1992).

(7) P. Adam, J. Janseky and An. V. Vinogradov, Optics. Comm. 80, 155 (1990).

[8] J. Janszky, P. A dam and An. V. Vinogradov, Phys. Rev. Lett. 68, 3816 (1992).

|9| P. Adam and J. Janseky, Phys. Lett. A 149, 67 (1990).

(10) P. Adam, J. Janszky and An. V. Vinogrador, Phys. Lett. A 160, (1991).

(11) J. Janszky, P. A dam, M. Bertolotti and C. Sibilia, Quantum Opt. 4, 163 (1992).

[12] D. T. Pegg and S. M. Barnett, Phys. Rev. A 39, 1665 (1990).

[13] W.Schleich, M. Pernigo and Fam Le Kien, Phys. Rev. A 44, 2172 (1991).

(14) J. Janszky and An. V. Vinogradow, Mol. Cryst. Liq. Cryst. Sci. Technol. - Sec. B, Nonlinear Opt. (in print).

[15] J. Janseky, P. Adam, An. V. Vinogradov and T. Kobayashi, Spectrochim. Acta 48A, 31 (1992). 